\title{
Creation of the horizontal-vertical illusion through imagery
}

\author{
BENJAMIN WALLACE \\ Cleveland State University, Cleveland, Ohio
}

Perception of the horizontal-vertical illusion was examined as a function of imaging ability. Subjects who scored either high or low on this attribute (as assessed by the Vividness of Visual Imagery Questionnaire, or VVIQ) were asked to image an inducing line or lines of the illusory stimulus. High imagers reported perceiving the illusion whether the inducing line(s) were imaged or were physically present. Also, the magnitude of the illusion as created by imagery-induced lines was equivalent to that produced for physically present lines. Low imagers reported perceiving the illusion only when inducing lines were physically present.

The substitution of an image for a physical stimulus to create a percept has recently been shown to be a possibility for a variety of phenomena. As examples, such substitution has been reported for perceptual adaptation to displacing prisms (Finke, 1979), letter search and identification (Podgorny \& Shepard, 1978), observation of the McCollough effect (Finke \& Schmidt, 1977, 1978; Kunen \& May, 1980), perceptual acuity of objects in the peripheral visual field (Finke \& Kosslyn, 1980), perception of area and contrast effects (Finke \& Kurtzman, 1981 ), production of the Poggendorff illusion (Goldstein \& Weintraub, 1973; Pressey \& Wilson, 1974), and creation of the autokinetic effect (Wallace, 1980).

Finke (1980) argued that not only can an image substitute for a physical stimulus, but also that the two are equivalent in their effect on perception. When such equivalence is considered for geometrical illusions, an image should substitute for the inducing element of the illusory figure. For example, in the Mueller-Lyer figure, images of the arrows and feathers should produce the Mueller-Lyer illusion as if they were physically present stimuli. And, in fact, this was demonstrated by Berbaum and Chung (1981).

The procedure of Berbaum and Chung (1981) can be employed easily with most geometrical illusions. However, with the horizontal-vertical illusion, the inducing stimulus can be either the horizontal line or the vertical line. Therefore, which line does an experimenter ask subjects to image in an investigation of the horizontalvertical illusion? The answer is that it may not matter, since either line may be capable of inducing the illusion. It is also possible that the illusion may be created by having subjects image both lines. The present investigation was conducted to probe these possibilities.

This research was supported by a Creative Activities and Research grant from Cleveland State University. Kathy Tomasko is gratefully acknowledged for her assistance with data collecting. Reprint requests should be sent to: Benjamin Wallace, Department of Psychology, Cleveland, Ohio 44115.

\section{METHOD}

\section{Subjects}

Forty volunteers from introductory psychology classes served as the subjects. All individuals previously had completed the Vividness of Visual Imagery Questionnaire, or VVIQ (Marks, 1973). This has been shown to be a good predictor of who is most capable of utilizing imagery in a perceptual task (Gur \& Hilgard, 1975). Half of the subjects were selected for scoring in the top one-third of those who had participated in a mass-testing session of 482 individuals. The remaining subjects were chosen for scoring in the lowest one-third of the respondents.
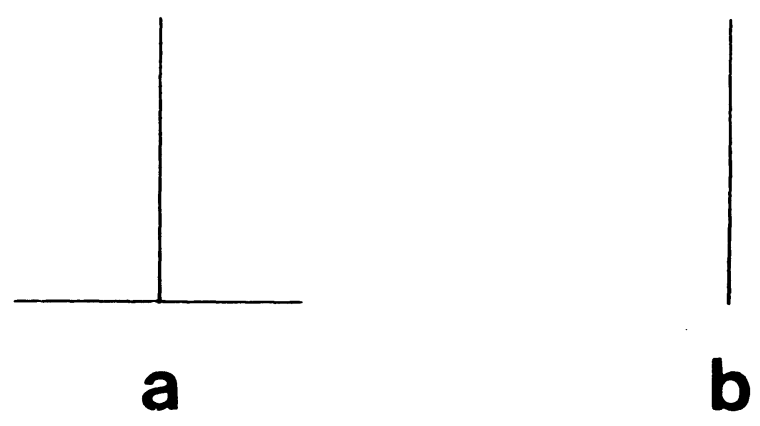

\section{C}

Figure 1. Horizontal-vertical figure stimuli. 


\section{Stimuli}

Four stimulus configurations, produced on white, nonglare paper, were employed (see Figure 1). For figures $1 \mathrm{a}, 1 \mathrm{~b}$, and $1 \mathrm{c}$, the line lengths were $12 \mathrm{~cm}$. Figure $1 \mathrm{~d}$ was physically nonexistent; the subjects were to imagine both the horizontal and vertical lines.

\section{Procedure}

Five high and five low imagers were assigned randomly to each of the four stimulus conditions. The subjects were tested individually by an experimenter who was unaware of an individual's VVIQ score and of the hypothesis of the experiment. When Figure 1b was presented, the subjects were first shown a horizontal line equal in length to the vertical line. The horizontal line was then removed, and the subjects were asked to form an image of the horizontal line and to place it with the visible, vertical line so as to form an inverted-T figure. To make certain the subjects understood the task assignment, the experimenter pointed to where the subjects should place their imaginary, horizontal line. When Figure 1c was presented, the protocols were identical to those employed for Figure $1 \mathrm{~b}$, except that the subjects were to imagine the vertical line and place it with the present horizontal line.

For Figure 1d, which was physically nonexistent, the subjects were shown simultaneously a horizontal line and a vertical line of equal length but not in an inverted-T format. These lines were then removed, and the subjects were told to form an inverted $T$ of the two lines. To maintain identical protocols with the other stimulus presentations, the experimenter pointed to where the subjects should produce their imaginary, horizontal and vertical, lines.

After the experimental manipulation for Figures $1 \mathrm{~b}, 1 \mathrm{c}$, and $1 \mathrm{~d}$, the subjects were asked to respond to one or more questions. First, they were asked, "Are you able to see the line(s) that I have asked you to imagine?" If the subjects responded "no," no further questions were asked. If the subjects responded "yes," they were asked, "Can you tell me if the horizontal line appears to be longer than the vertical line, if the vertical line appears to be longer than the horizontal line, or if both lines appear to be equal in length?" (The order of stated alternatives was randomized.) If the subjects responded that the lines appeared to be equal in length, this ended questioning by the experimenter. If the subjects responded that one line appeared to be longer, they were asked, "By how much does the horizontal/vertical line appear to be longer?" The subjects were given a ruler, calibrated in millimeters, to make this estimate. Also, no clues concerning what their response should be were given to the subjects.

For Figure 1a, which did not require the deployment of imagery, the subjects were first asked to respond to the second question as stated above. If they indicated that one line appeared to be longer, they were to answer the third question as stated above. After participating in the experiment, the subjects were asked if they had ever seen the figure to which they had been exposed. One indicated that he had for Figure 1a but had not known it was a visual illusion.

\section{RESULTS AND DISCUSSION}

Subjects judged to be high in imagery ability responded to the various figures quite differently from the way the low imagers responded. Of the 20 high imagers, all correctly reported, regardless of figure type, the horizontal-vertical illusion in which the vertical line is perceived as being longer than the horizontal line. Low imagers, for the most part, were incapable of imaging lines and as a result did not experience the horizontalvertical illusion. However, when imagery was not required, as for Figure 1a, all five low imagers correctly reported the illusion.
Table 1

Magnitude Estimates (in Millimeters) of the Horizontal-Vertical Illusion as a Function of Imagery Ability and Figure Type

\begin{tabular}{rlrrrr}
\hline & & \multicolumn{4}{c}{ Figure Type } \\
\cline { 2 - 6 } Imagery Ability & \multicolumn{1}{c}{$1 \mathrm{a}$} & \multicolumn{1}{c}{$1 \mathrm{~b}$} & \multicolumn{1}{c}{$1 \mathrm{c}$} & \multicolumn{1}{c}{$1 \mathrm{~d}$} \\
\hline \multirow{2}{*}{ High } & Mean & 19.00 & 20.80 & 21.80 & 21.00 \\
& SD & 2.24 & 2.77 & 3.19 & 3.00 \\
\multirow{2}{*}{ Low } & Mean & 19.00 & $18.00^{*}$ & $20.50^{*}$ & \\
& SD & 1.58 & & & \\
\hline
\end{tabular}

*The horizontal-vertical illusion for a single subject.

For high imagers, the magnitude estimates (see Table 1) for the various conditions were not statistically different from each other (all $t$ values were less than 1.00). That is, the horizontal-vertical illusion was present, and by an equivalent amount, for all conditions. For low imagers, the horizontal-vertical illusion was equal to that reported for high imagers for Figure 1a.

The high imagers appeared better able to produce stimuli via imagery and subsequently to employ these stimuli in the creation of a visual illusion. Also, regardless of which configuration the high imagers were shown, the illusion was clearly there. This was not true for low imagers. Thus, at least for high imagers, this experiment further supported the equivalence of an image and a physical stimulus (Finke, 1980) in the creation of a visual illusion. Furthermore, it does not appear to matter which line is employed as the inducing stimulus. In fact, both lines can be imagined to ultimately produce the horizontal-vertical illusion.

Also, if equivalence exists between imagined lines and physically present lines in the creation of the horizontalvertical illusion, this might imply that the same information-processing mechanisms might be operating when events are perceived through the assistance of imagery as during the perception of an event with the presence of physical stimuli. However, the horizontal-vertical illusion has been explained most often as being the result of the orientation of lines on the retina (Avery \& Day, 1969; Kuennapas, 1958). This clearly cannot be the case with inducing lines created through imagery. Yet, the illusion was still present. Therefore, mechanisms beyond the retina must be playing a role in the production of the horizontal-vertical illusion. The identity and nature of these mechanisms are not known, and further research must be conducted before a theoretical explanation of the present results can be proposed.

\section{REFERENCES}

Avery, G. C., \& Day, P. H. (1969). Basis of the horizontalvertical illusion. Journal of Experimental Psychology, 81, 376-380.

Berbaum, K., \& Chung, C. P. (1981). Mueller-Lyer illusion induced by imagination. Journal of Mental Imagery, 5, 125-128.

Finke, R. A. (1979). The functional equivalence of mental images. and errors of movement. Cognitive Psychology, 11, 235-264.

Finke, R. A. (1980). Levels of equivalence in imagery and perception. Psychological Review, 87, 113-132.

Finke, R. A., \& Kosslyn, S. M. (1980). Mental imagery acuity in 
the peripheral visual field. Journal of Experimental Psychology: Human Perception and Performance, 6, 126-139.

Finke, R. A., \& Kurtzman, H. S. (1981). Area and contrast effects upon perceptual and imagery acuity. Journal of Experimental Psychology: Human Perception and Performance, 7, 825-832.

Finke, R. A., \& Schmidt, M. J. (1977). Orientation-specific color aftereffects following imagination. Journal of Experimental Psychology: Human Perception and Performance, 3, 599-606.

Finke, R. A., \& SchmidT, M. J. (1978). The quantitative measure of pattern representation in images using orientation-specific color aftereffects. Perception \& Psychophysics, 23, 515-520.

Goldstein, M. B., \& Weintraub, D. J. (1973). The parallelless Poggendorff: Virtual contours put the illusion down but not out. Perception \& Psychophysics, 11, 353-354.

Gur, R. C., \& Hilgard, E. R. (1975). Visual imagery and the discrimination of differences between altered pictures simultaneously and successively presented. British Journal of Psychology, 66, 341-345.
KUENNAPAS, T. M. (1958). Influence of head inclination on the vertical-horizontal illusion. Journal of Psychology, 46, 179-185.

Kunen, S., \& MaY, J. G. (1980). Spatial frequency content of visual imagery. Perception \& Psychophysics, 28, 555-559.

MARKs, D. F. (1973). Visual imagery differences in the recall of pictures. British Journal of Psychology, 64, 17-24.

Podgorny, P., \& Shepard, R. N. (1978). Functional representations common to visual perception and imagination. Journal of Experimental Psychology: Human Perception and Performance, 4, 21-35.

Pressey, A. W., \& Wilson, A. E. (1974). The Poggendorff illusion in imagination. Bulletin of the Psychonomic Society, 3, 477-449.

WALlaCe, B. (1980). Autokinetic movement of an imagined and an hypnotically-hallucinated stimulus. International Journal of Clinical and Experimental Hypnosis, 28, 386-393.

(Manuscript received for publication October 24, 1983.) 